\title{
Calcium Pyrophosphate And Monosodium Urate Activate The NLRP3 Inflammasome Within Bladder Urothelium Via Reactive Oxygen Species And TXNIP
}

This article was published in the following Dove Press journal:

Research and Reports in Urology

\author{
Shelby $N$ Harper $\mathbb{D}^{1,2}$ \\ Patrick D Leidig ${ }^{2}$ \\ Francis $M$ Hughes Jr (D) $^{2}$ \\ Huixia Jin (iD ${ }^{2}$ \\ J Todd Purves (iD) 2,3 \\ 'Duke University School of Medicine, \\ Durham, NC, USA; ${ }^{2}$ Department of \\ Surgery, Division of Urology, Duke \\ University Medical Center, Durham, NC, \\ USA; ${ }^{3}$ Department of Pediatrics, Duke \\ University Medical Center, Durham, \\ NC, USA
}

\begin{abstract}
Objective: To investigate the in vitro activation of the NLRP3 inflammasome within bladder urothelium by stone-forming components. Further, to describe the contributions of reactive oxygen species (ROS) and thioredoxin-interacting protein (TXNIP), an important structural component of the inflammasome, to this activation.

Methods: Urothelial cells were harvested and incubated overnight. For agonist studies, cells were treated with varying concentrations of calcium pyrophosphate (CPPD) and monosodium urate (MSU). For inhibitor studies, cells were treated with either $\mathrm{N}$-acetylcysteine (NAC) (1 hr) or Verapamil (4 hrs) prior to incubation with either CPPD $(62.5 \mathrm{ug} / \mathrm{mL})$ or MSU (1.25 ug/mL) for $24 \mathrm{hrs}$. Untreated controls were incubated with ATP (1.25 mM) for 1 hr to maximally stimulate NLRP3 inflammasome activity (measured as caspase-1 cleavage of the fluorogenic substrate Ac-YVAD-AFC). Results are reported as a percentage of maximum ATP response.

Results: CPPD and MSU activate caspase-1 in urothelial cells in a dose-dependent manner, reaching $\sim 50 \%$ and $\sim 25 \%$ of the ATP response, respectively. Pre-treatment with the general ROS scavenger NAC reduces this activation in a dose-dependent manner. Additionally, activation was suppressed through treatment with Verapamil, a known downregulator of TXNIP expression.

Conclusion: The stone components CPPD and MSU activate NLRP3 in an ROS and TXNIP-dependent manner in bladder urothelium. These findings demonstrate the importance of ROS and TXNIP, and suggest that targeting either may be a way to decrease stonedependent NLRP3 inflammation within the bladder.
\end{abstract}

Keywords: stones, urothelium, inflammation, NLRP3 inflammasome, TXNIP, ROS

\section{Introduction}

Urine contains many noxious chemical moieties, such as organic acids and salts, which can irritate or inflame the lumen of the urinary tract. In patients who are particularly sensitive to these, including those who have defects in the protective glycosaminoglycan (GAG) layer, they can cause urinary symptoms of urgency and frequency or even pain. The most potent forms of these irritants are the components that can crystallize into urinary stones. Once they have crystallized to a sufficiently large size, they are capable of mechanically breaching the protective GAG layer to expose the vulnerable urothelium below. Perhaps the most extreme form of this
Correspondence: Francis M Hughes J Department of Surgery, Division of Urology, Duke University Medical Center, P.O. Box 383I, Durham, NC 277I0, USA $\mathrm{Tel}+$ I 8437092128

Email monty.hughes@duke.edu 
irritation is seen when stone material becomes impacted in the ureter and can cause intense pain from renal colic and local inflammation. Moreover, impacted ureteral stones produce local fibrosis and are the most common known cause of ureteral strictures that can cause long-term morbidity and need for surgical intervention. ${ }^{1,2}$ Exactly how these chemical urinary components interface with the urothelium to provoke functional disturbances, fibrosis, and pain is not completely understood.

The consequences of urothelial exposure to noxious chemical stimuli are thought to potentially result from inflammation. Recent studies of inflammation in innate immune cells and epithelial tissues have identified a supramolecular structure, known as the inflammasome, as being critically important in mediating this inflammatory response. ${ }^{3-5}$ By far the best studied of these is the NLRP3 inflammasome. In fact, our own lab has highlighted the central importance of NLRP3 in triggering inflammation within bladder urothelium in different pathologic states. ${ }^{6-10}$ The NLRP3 inflammasome is comprised, in part, of the nod-like receptor NLRP3, a structural co-factor protein called thioredoxininteracting protein (TXNIP), and the adaptor protein ASC. In the presence of activating stimuli, this complex forms, recruits, and activates caspase-1, resulting in the cleavage and maturation of the pro-inflammatory cytokines IL- $1 \beta$ and IL-18. These cytokines are released from the cell via a form of necrotic cell death called pyroptosis, where they go on to promote inflammation.

There are a number of known activators of the inflammasome, including pathogen associated molecular patterns (PAMPs) and damage associated molecular patterns (DAMPs). ${ }^{11}$ Although many classes of inflammasome can respond to PAMPS, the NLRP3 inflammasome is by far the most well-known to respond to DAMPs. ${ }^{11}$ In studies of gout and pseudogout, ${ }^{12}$ calcium pyrophosphate (CPPD) and monosodium urate (MSU), two major components of urinary stones, have been shown to act as DAMPs to stimulate NLRP3 inflammasome activity in macrophages. In similar studies of osteoarthritis, both CPPD and MSU promote inflammation mediated by this inflammasome. ${ }^{13}$ In their role as DAMPs, these two components are thought to potentiate NLRP3 inflammasome activation by stimulating intracellular reactive oxygen species (ROS) production. Under normal conditions, TXNIP, a structural co-factor of the assembled inflammasome, is bound to the cellular antioxidant thioredoxin. When ROS are produced, they oxidize thioredoxin, resulting in the dissociation of TXNIP.
Free TXNIP is then able to bind to NLRP3 to promote formation of the active inflammasome. While ROSmediated NLRP3 activation, and the importance of TXNIP, have been explored in other cell types, ${ }^{14,15}$ its role in stone-mediated urothelial inflammation is not yet defined. In this study, we investigated the potential of this pathway in the urothelial response to urinary stone components and identified potential targets to mitigate the subsequent inflammation.

\section{Materials And Methods}

\section{Animals}

All protocols adhered to the NIH Guide for the Care and Use of Laboratory Animals and were approved by the Institutional Animal Care and Use committee at Duke University Medical Center. Female Sprague Dawley rats ( $\approx 200$ grams, 40-50 days of age, Envigo, Indianapolis, IN) were used in all studies.

\section{Cell Isolation}

The cell isolation protocol was modified from a previously published method. ${ }^{6,16}$ Briefly, rats were sacrificed and the bladders removed and placed in sterile PBS. Bladders were then inverted over an 18-gauge blunt tip needle, inflated with PBS, and a purse string suture was used to tie off the bladder. The inflated bladder was then submerged in Collagenase $\mathrm{P}(1 \mathrm{mg} / \mathrm{mL}$ in complete media) and shaken for $1 \mathrm{hr}$ at $37^{\circ} \mathrm{C}$. Cells were then passed through a $40 \mu \mathrm{m}$ nylon mesh to remove debris, pelleted and resuspended in complete media $[\mathrm{F}-12 \mathrm{~K}$ media (HyClone Laboratories, Logan, UT) supplemented with $10 \%$ low-endotoxin dialyzed fetal bovine serum (HyClone Laboratories, Logan, UT), $10 \mu \mathrm{M}$ nonessential amino acids (HyClone Laboratories, Logan, UT), $\quad 1.0 \mu \mathrm{g} / \mathrm{mL}$ hydrocortisone (Sigma-Aldrich, St. Louis, MO), $10 \mu \mathrm{g} / \mathrm{mL}$ insulin (Gibco Laboratories, Gaithersburg, Maryland), $5 \mu \mathrm{g} / \mathrm{mL}$ transferrin (Gibco Laboratories, Gaithersburg, MD), $6.7 \mathrm{ng} / \mathrm{mL}$ selenium (Gibco Laboratories, Gaithersburg, MD), $100 \mathrm{U} / \mathrm{mL}$ penicillin (Gibco Laboratories, Gaithersburg, MD), and 100 $\mu \mathrm{g} / \mathrm{mL}$ streptomycin (Gibco Laboratories, Gaithersburg, MD)]. Cells were counted and plated at 50,000 cells/well in $90 \mu \mathrm{L}$ complete media in black-walled 96-well plates. Cells were then incubated in a water-saturated environment for $24 \mathrm{hrs}$ at $37^{\circ} \mathrm{C}, 95 \%$ air, and $5 \% \mathrm{CO}_{2}$. Media was removed and replaced with fresh media $(90 \mu \mathrm{L}$ for 
agonist studies, $80 \mu \mathrm{L}$ for inhibitor studies) just prior to the start of experimental treatments.

\section{Experimental Treatments}

In vitro experiments were performed essentially as previously described. ${ }^{6}$ For agonist response studies, CPPD and MSU (InvivoGen, San Diego, CA) were prepared to the stock concentrations of $1.25 \mathrm{mg} / \mathrm{mL}$ and $12.5 \mu \mathrm{g} / \mathrm{mL}$, respectively. Using PBS (for CPPD) or complete media (for MSU), 1:2 serial dilutions were prepared. Wells were then treated with $10 \mu \mathrm{L}$ of the appropriate dilution of stone DAMP for a final volume of $100 \mu \mathrm{L}$. After treatment, cells were incubated for another $24 \mathrm{hrs}$ at $37^{\circ} \mathrm{C}$.

For inhibition response studies, the general ROS scavenger $\mathrm{N}$-acetylcysteine was prepared to the stock concentration of $5 \mathrm{mM}$ for MSU treatment and to $50 \mathrm{mM}$ for CPPD treatment. Prior to treatment, the stock concentration of NAC was buffered to $\mathrm{pH}$ 7.2. Verapamil was prepared to a stock concentration of $1.5 \mathrm{mM}$ for serial dilution and cell treatment. Using complete media for NAC and PBS for Verapamil, 1:2 serial dilutions were prepared. Cells were treated with $10 \mu \mathrm{L}$ of NAC or Verapamil and incubated at $37^{\circ} \mathrm{C}$ for 1 or $4 \mathrm{hrs}$, respectively. After pre-treatment, cells were then treated with 10 $\mu \mathrm{L}$ of CPPD (62.5 $\mu \mathrm{M}$ final) or MSU (1.25 $\mu \mathrm{M}$ final) to a final volume of $100 \mu \mathrm{L}$. Plates were then incubated for an additional $24 \mathrm{hrs}$ at $37^{\circ} \mathrm{C}$. One hour prior to the end of the incubation, untreated control wells were administered $1.25 \mathrm{mM}$ ATP to serve as a standard for maximal caspase response.

\section{Caspase-I Assay}

The caspase- 1 assay was performed as reported. ${ }^{17}$ Briefly, media were removed and cells lysed in $50 \mu \mathrm{L}$ lysis buffer (10 $\mathrm{mM} \mathrm{MgCl}_{2}$ and $0.25 \%$ Igepal CA-630) for 5 mins. An additional $50 \mu \mathrm{L}$ of storage buffer (40 mM HEPES ( $\mathrm{pH}$ 7.4), $20 \mathrm{mM} \mathrm{NaCl}, 2 \mathrm{mM}$ EDTA and $20 \%$ glycerol) was added, and the plates frozen at $-80^{\circ} \mathrm{C}$ until used $(>30$ mins). Plates were then thawed and $50 \mu \mathrm{L}$ of $50 \mathrm{mM}$ Hepes with $10 \%$ Sucrose and $0.1 \%$ CHAPS, $10 \mu \mathrm{L}$ dithiothreitol (final concentration of $5.5 \mathrm{mM}$ ), and $20 \mu \mathrm{L}$ Z-YVAD-AFC substrate (final concentration of $110 \mu \mathrm{M}$ ) were added to each well. Plates were then incubated in the dark for $1 \mathrm{hr}$ at $37^{\circ} \mathrm{C}$ with mild shaking. Florescence was then measured (excitation $400 \mathrm{~nm}$, emission $505 \mathrm{~nm}$ ). Florescence in untreated wells ( $0 \mathrm{mM}$ stone DAMP) was subtracted from all wells and results normalized to the
ATP response. Results were reported as a percentage of ATP response.

\section{Statistical Analysis}

Statistical analysis was performed by a one-way analysis of variance followed by a Dunnett's post-hoc analysis using GraphPad InStat software (La Jolla, CA).

\section{Results CPPD And MSU Produce A Dose-Dependent Increase In Caspase-I Activation}

CPPD triggers a robust and dose-dependent activation of caspase-1 in isolated urothelium in vitro, with a maximal response of approximately $50 \%$ of the ATP response and an $\mathrm{EC}_{50}$ of $62.5 \mu \mathrm{g} / \mathrm{mL}$ (Figure 1A). MSU was less efficacious yet more potent than CPPD, with a maximal response of $25 \%$ of the $\mathrm{ATP}$ response but an $\mathrm{EC}_{50}$ of $0.156 \mu \mathrm{g} / \mathrm{mL}$ (Figure 1B).

\section{N-Acetylcysteine Inhibits Caspase-I Activation In Cells Treated With Stone DAMPs}

To determine if CPPD and MSU signal for inflammasome activation in the urothelia through ROS, we utilized the general ROS scavenger NAC. As shown in Figure 2A, a NAC concentration of $5 \mathrm{mM}$ was sufficient to completely suppress caspase-1 activation in cells treated with $\mathrm{CPPD}\left(\mathrm{IC}_{50}=625 \mu \mathrm{M}\right)$. However, as shown in Figure 2B, a NAC concentration of just $500 \mu \mathrm{M}$ was sufficient to completely suppress caspase-1 activation in MSU-treated cells $\left(\mathrm{IC}_{50}=31.25 \mu \mathrm{M}\right)$.

\section{Verapamil Inhibits Caspase-I Activation}

Verapamil is a calcium channel blocker that has been shown to downregulate the expression of the NLRP3 binding protein TXNIP, ${ }^{18,19}$ and thus has been used in several studies to assess a role for this critical structural component of the NLRP3 inflammasome in DAMP pathways mediated by ROS. ${ }^{20,21}$ As shown in Figure 3A, a dose of $150 \mu \mathrm{M}$ Verapamil was sufficient to completely inhibit CPPD-mediated caspase- 1 activation $\left(\mathrm{IC}_{50}=100 \mu \mathrm{M}\right)$. In a similar fashion, shown in Figure 3B, MSU-induced caspase-1 activation was also completely suppressed in cells by $150 \mu \mathrm{M}$ Verapamil $\left(\mathrm{IC}_{50}=100 \mu \mathrm{M}\right)$. 

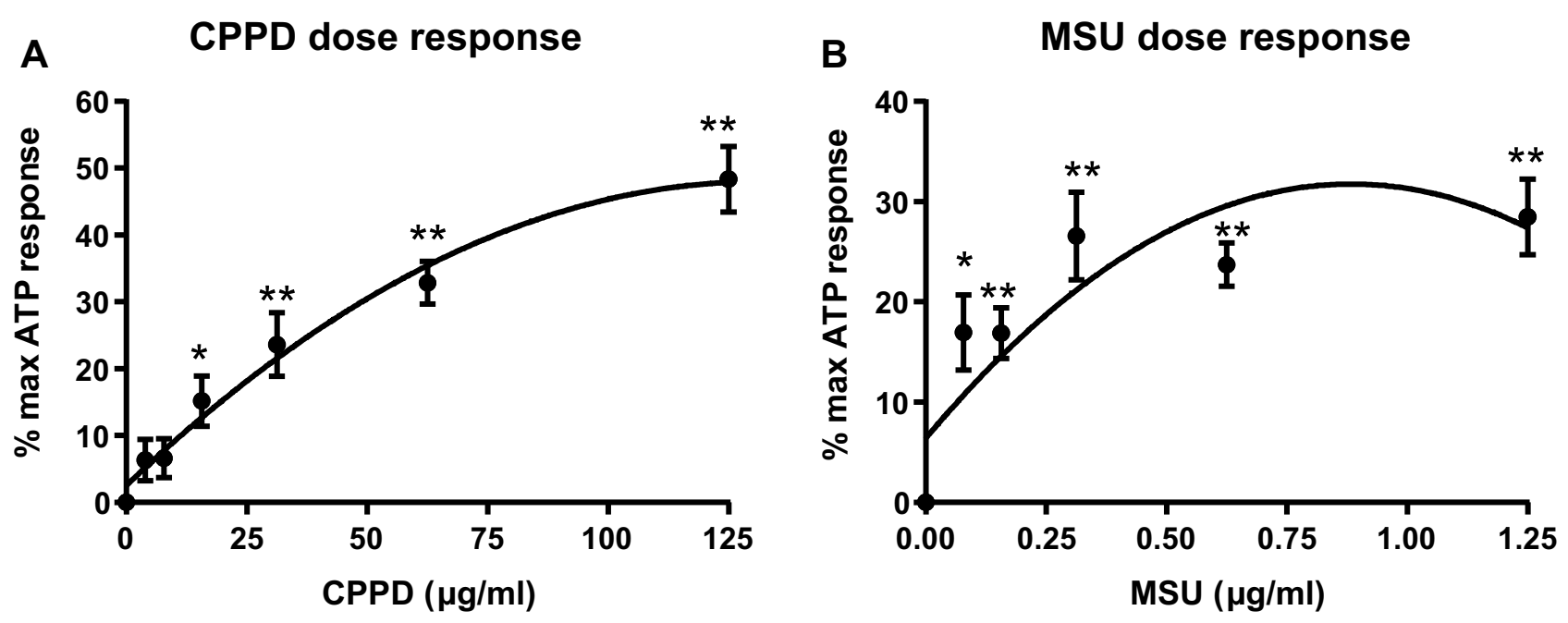

CPPD: calcium pyrophosphate

MSU: monosodium urate

Figure I The stone DAMPs CPPD and MSU activate caspase-I in a dose-dependent manner. Urothelial cells were incubated overnight prior to treatment with either CPPD (A) or MSU (B) for 24 hrs. Additional wells were treated with $1.25 \mathrm{mM}$ ATP for I hr to indicate maximal caspase-I activation and DAMP-treated wells were normalized to these ATP-treated wells. $\mathrm{n}=9$ for all doses of CPPD and MSU. ${ }^{*} \mathrm{p}<0.05$; ${ }^{* *} \mathrm{p}<0.0 \mathrm{l}$ by one-way ANOVA and Dunnett's post-hoc test.
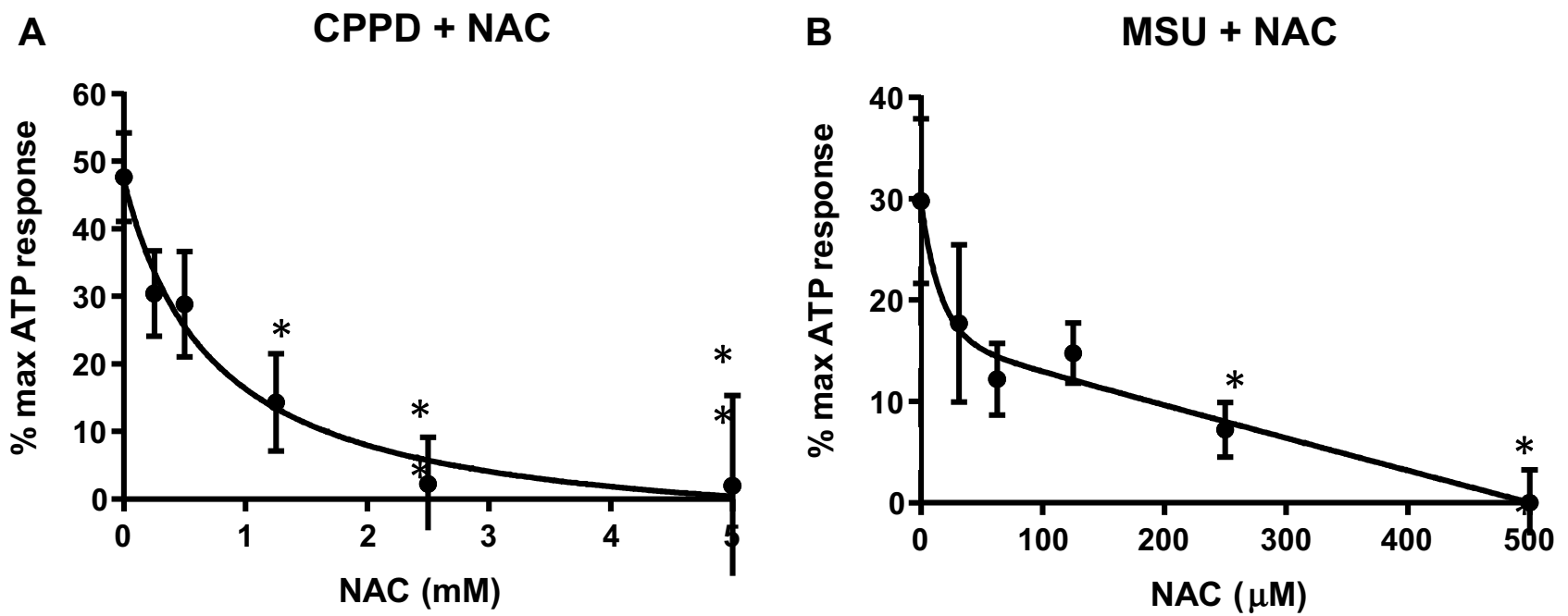

CPPD: calcium pyrophosphate

MSU: monosodium urate

NAC: N-acetylcysteine

Figure 2 NAC inhibits caspase-I activation in cells treated with CPPD or MSU. Urothelial cells were incubated overnight and then treated with decreasing concentrations of NAC for I hr before treatment with $62.5 \mu \mathrm{g} / \mathrm{mL}$ CPPD (A) or I.25 $\mu \mathrm{g} / \mathrm{mL}$ MSU (B) for $24 \mathrm{hrs}$. The caspase-I assay was then performed as described in the Materials and Methods section. CPPD-treated cells had a higher IC $50(625 \mu \mathrm{M})$ versus MSU-treated cells $\left(\mathrm{IC}_{50}=31.25 \mu \mathrm{M}\right)$. $\mathrm{n}=9$ for all NAC treatment doses for both CPPD and MSU. $*_{p}<0.05$ by one-way ANOVA and Dunnett's post-hoc test.

\section{Discussion}

This study provides the first exploration into the urinary DAMP-ROS-NLRP3 inflammasome pathway within the urothelium. We found that NLRP3 is activated in vitro in urothelial cells by two common stone-forming components. Importantly, we also found that it is mediated through an upregulation in intracellular ROS and release of TXNIP from thioredoxin. Specifically, the general ROS scavenger NAC was able to prevent inflammasome activation in both CPPD and MSU-treated urothelial cells. Further, directed targeting of a ROS-responsive protein (TXNIP) that forms a structural component of the 

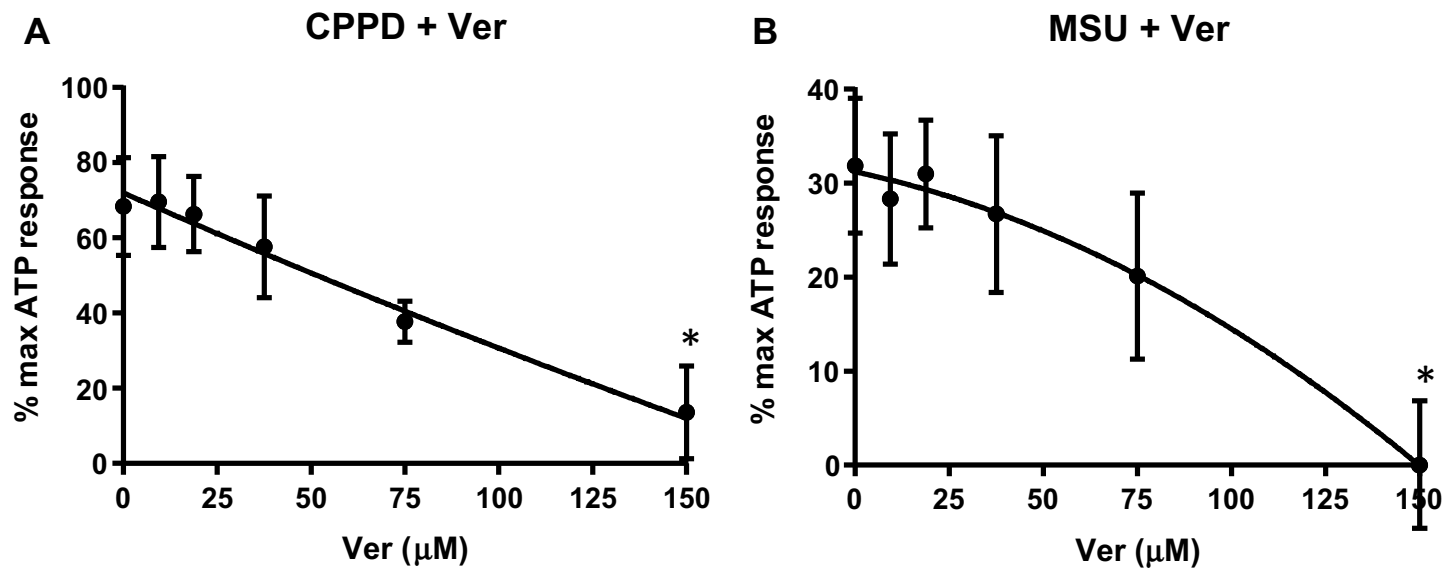

CPPD: calcium pyrophosphate MSU: monosodium urate Ver: verapamil

Figure 3 Verapamil suppresses caspase-I activation. Urothelial cells were incubated overnight and then treated with decreasing concentrations of Verapamil for 4 hrs before treatment with $62.5 \mu \mathrm{g} / \mathrm{mL}$ CPPD (A) or $1.25 \mu \mathrm{g} / \mathrm{mL} \mathrm{MSU}$ (B) for $24 \mathrm{hrs}$. The caspase-I assay was then performed as described in the Materials and Methods section. Both CPPD and MSU-treated cells had the same $I_{50}(100 \mu M)$. $n=10$ for all doses of Verapamil and CPPD treated wells; $n=5$ for all doses of Verapamil and MSU treated wells. $*_{p}<0.05$ by one-way ANOVA and Dunnett's post-hoc test.

NLRP3 inflammasome was also able to prevent inflammasome activity. These findings demonstrate a ROS-driven pathway in stone-induced urothelial inflammation that relies on the TXNIP protein for functionality. Given the consequences of inflammation within the urinary tract, understanding the nuances of this pathway within the urothelium is critical to understanding how to target it clinically in patients.

Interestingly, while NAC and Verapamil both were able to abolish inflammasome activation by CPPD and MSU, we found exciting differences in their respective abilities to do so. First, NAC doses required to completely inhibit inflammasome activation were higher in cells treated with CPPD $(5 \mathrm{mM})$ compared to $\mathrm{MSU}$ $(500 \mu \mathrm{M})$. This differential response is possibly the result of differences in intracellular ROS production by the various stone DAMPs. Second, there were no differences in treatment dose of Verapamil required to completely inhibit inflammasome activation in either CPPD or MSU-treated cells. Maximal inhibition of caspase-1 activity was seen at a Verapamil dose of $150 \mu \mathrm{M}$ after treatment with either stone DAMP. Therefore, it appears that functional TXNIP is required for the activation of NLRP3 regardless of the magnitude of ROS production. Based on these findings, targeted therapies aimed at impeding different steps within this pathway may be useful to inhibit stonemediated inflammasome activity.
$\mathrm{NAC}$ is an FDA-approved medication that functions as a general ROS scavenger and acts as a precursor molecule for the regeneration of intracellular glutathione, another scavenger of ROS. ${ }^{22}$ Clinically, NAC is very effective in a number of patient populations, such as acetaminophen overdose, polycystic ovarian syndrome, and chronic bronchitis. While the usefulness of NAC in these patient populations is wellestablished, its potential in treating urinary DAMP-mediated bladder inflammation has never before been explored. Our findings suggest that this drug may be a useful tool in turning off bladder inflammation caused by urinary DAMPs, thereby allowing for reduction in inflammatory complications.

Verapamil is a non-dihydropyridine calcium channel blocker used in a number of medical conditions, such as hypertension, angina, and cluster headaches. An early study suggested that its calcium channel blocking ability might reduce the level of stone forming components in the urine, but a subsequent study did not corroborate this finding. ${ }^{23,24}$ However, in this study, verapamil's ability to downregulate expression of TXNIP ${ }^{25,26}$ proved a useful tool to block stone-DAMP activation of NLRP3. Therefore, while it may not affect the concentration of stone forming moieties, it may actually protect the urinary tract from these pro-inflammatory urinary components.

This study demonstrates that urinary stone-forming DAMPS activate NLRP3 in urothelial cells via ROS production and require the presence of TXNIP. This pathway is effectively blocked by the administration of the anti- 
oxidant, NAC, or by down-regulating TXNIP expression with verapamil. These agents may be useful in preventing lower urinary tract inflammation, pain, and risk of fibrosis and scarring in stone forming patients. More broadly, and the subject of future studies, many other urinary DAMPs could also activate the NLRP3 inflammasome and provoke a urothelial inflammatory response via the same pathway demonstrated in this investigation. It is well-known that consumption of certain foods, such as chocolate, alcohol, and acidic juices, can exacerbate lower urinary tract symptoms in sensitive patients. Abstinence from these dietary factors is very effective, but that requires the identification of the specific item which can be challenging. If the pathway elucidated here is common to many other urinary DAMPs, then targeting ROS production, TXNIP expression and/or NLRP3 activation could be implemented as a treatment strategy even if the inciting factor is unknown. Additionally, we have shown in a prior study that diabetic metabolites are capable of activating NLRP3 in urothelium, and this contributes to the development of diabetic bladder dysfunction (DBD). ${ }^{6}$ If future studies demonstrate that these metabolites have the same mechanism of action as the stone DAMPs (uric acid being an example of both) then targeting ROS production or TXNIP expression may be useful in preventing DBD.

\section{Conclusion}

Urinary stones are comprised of components, such as calcium pyrophosphate and monosodium urate, that promote NLRP3 inflammasome activation in vitro within urothelial cells. The present study suggests that urinary stone components drive the activation of the NLRP3 inflammasome by increased intracellular concentrations of ROS that impinge upon TXNIP. Importantly, this study illuminates steps in the pathway that can be pharmacologically impeded through use of $\mathrm{N}$-acetylcysteine and verapamil. Further studies are necessary in vivo to determine the ability of urinary stone components to promote inflammation through NLRP3 and trigger significant inflammatory complications. After such studies, we can then begin to assess the therapeutic potential of these two medications in stone disease.

\section{Acknowledgments}

This work was awarded a Herbert Brendler, MD 2019 Urology Care Foundation Summer Medical Student Fellowship from the American Urological Association, as well as the Poindexter Fellowship from Duke University School of Medicine. This research was supported by the
National Institute of Diabetes and Digestive and Kidney Diseases of the National Institutes of Health, USA (Award Numbers R01DK103534 and R01DK117890 to JTP) and intramural funds from Duke University Medical Center, Department of Surgery, Division of Urology. The abstract of this paper was presented at the Multidisciplinary Benign Urology Research Day, the American Urological Association National Meeting, the Southeastern Section of the American Urological Association, the Kimbrough Urological Meeting, and the Society for Pelvic Research as a poster presentation or talk with interim findings. The AUA National Meeting poster abstract was published in the "2018 Annual Meeting Program Abstracts Supplement" in The Journal of Urology: https://doi.org/10.1016/j.juro.2018.02.773.

\section{Disclosure}

Dr Francis Hughes reports a patent 62/850015 pending to Duke University. The authors report no other conflicts of interest in this work.

\section{References}

1. Dong H, Peng Y, Li L, Gao X. Prevention strategies for ureteral stricture following ureteroscopic lithotripsy. Asian J Urol. 2018;5 (2):94-100. doi:10.1016/j.ajur.2017.09.002

2. Roberts WW, Cadeddu JA, Micali S, Kavoussi LR, Moore RG. Ureteral stricture formation after removal of impacted calculi. J Urol. 1998;159(3):723-726. doi:10.1016/S0022-5347(01)63711-X

3. Savage CD, Lopez-Castejon G, Denes A, Brough D. NLRP3inflammasome activating DAMPs stimulate an inflammatory response in glia in the absence of priming which contributes to brain inflammation after injury. Front Immunol. 2012;3:288. doi:10. 3389/fimmu.2012.00288

4. Rheinheimer J, de Souza BM, Cardoso NS, Bauer AC, Crispim D. Current role of the NLRP3 inflammasome on obesity and insulin resistance: a systematic review. Metabolism. 2017;74:1-9. doi:10.10 16/j.metabol.2017.06.002

5. Liu D, Zeng X, Li X, Mehta JL, Wang X. Role of NLRP3 inflammasome in the pathogenesis of cardiovascular diseases. Basic Res Cardiol. 2018;113(1):5. doi:10.1007/s00395-017-0663-9

6. Hughes FM Jr., Hirshman NA, Inouye BM, et al. NLRP3 promotes diabetic bladder dysfunction and changes in symptom-specific bladder innervation. Diabetes. 2018;68:430-440.

7. Hughes FM Jr., Vivar NP, Kennis JG, et al. Inflammasomes are important mediators of cyclophosphamide-induced bladder inflammation. Am J Physiol Renal Physiol. 2014;306(3):F299-308. doi:10.1152/ajprenal.00297.2013

8. Hughes FM Jr., Hill HM, Wood CM, et al. The NLRP3 inflammasome mediates inflammation produced by bladder outlet obstruction. J Urol. 2016;195:1598-1605. doi:10.1016/j.juro.2015.12.068

9. Hughes FM Jr., Kennis JG, Youssef MN, Lowe DW, Shaner BE, Purves JT. The NACHT, LRR and PYD domains-containing protein 3 (NLRP3) inflammasome mediates inflammation and voiding dysfunction in a lipopolysaccharide-induced rat model of cystitis. J Clin Cell Immunol. 2016;7(1). doi:10.4172/2155-9899.1000396

10. Inouye BM, Hughes FM Jr., Sexton SJ, Purves JT. The emerging role of inflammasomes as central mediators in inflammatory bladder pathology. Curr Urol. 2018;11(2):57-72. doi:10.1159/000447196 
11. Petrilli V, Dostert C, Muruve DA, Tschopp J. The inflammasome: a danger sensing complex triggering innate immunity. Curr Opin Immunol. 2007;19(6):615-622. doi:10.1016/j.coi.2007.09.002

12. Martinon F, Petrilli V, Mayor A, Tardivel A, Tschopp J. Goutassociated uric acid crystals activate the NALP3 inflammasome. Nature. 2006;440(7081):237-241. doi:10.1038/nature04516

13. Campillo-Gimenez L, Renaudin F, Jalabert M, et al. Inflammatory potential of four different phases of calcium pyrophosphate relies on NF-kappaB activation and MAPK pathways. Front Immunol. 2018;9:2248. doi:10.3389/fimmu.2018.02248

14. Joshi S, Wang W, Peck AB, Khan SR. Activation of the NLRP3 inflammasome in association with calcium oxalate crystal induced reactive oxygen species in kidneys. J Urol. 2015;193(5):1684-1691. doi:10.1016/j.juro.2014.11.093

15. Minutoli L, Puzzolo D, Rinaldi M, et al. ROS-mediated NLRP3 inflammasome activation in brain, heart, kidney, and testis ischemia/reperfusion injury. Oxid Med Cell Longev. 2016;2016:2183026. doi:10.1155/2016/2183026

16. Kloskowski T, Uzarska M, Gurtowska N, et al. How to isolate urothelial cells? Comparison of four different methods and literature review. Hum Cell. 2014;27(2):85-93. doi:10.1007/s13577-013-0070-y

17. Hughes FM Jr., Turner DP, Purves JT. The potential repertoire of the innate immune system in the bladder: expression of pattern recognition receptors in the rat bladder and a rat urothelial cell line (MYP3 cells). Int Urol Nephrol. 2015;47(12):1953-1964. doi:10.1007/s11255-015-1126-6

18. Melone MAB, Dato C, Paladino S, et al. Verapamil inhibits Ser202/Thr205 phosphorylation of tau by blocking TXNIP/ROS/ p38 MAPK pathway. Pharm Res. 2018;35(2):44. doi:10.1007/ s11095-017-2276-2
19. Xu G, Chen J, Jing G, Shalev A. Preventing beta-cell loss and diabetes with calcium channel blockers. Diabetes. 2012;61 (4):848-856. doi:10.2337/db11-0955

20. Abais JM, Xia M, Li G, et al. Nod-like receptor protein 3 (NLRP3) inflammasome activation and podocyte injury via thioredoxin-interacting protein (TXNIP) during hyperhomocysteinemia. J Biol Chem. 2014;289(39):27159-27168. doi:10.1074/jbc.M114.567537

21. Xu L, Lin X, Guan M, Zeng Y, Liu Y. Verapamil attenuated prediabetic neuropathy in high-fat diet-fed mice through inhibiting TXNIP-mediated apoptosis and inflammation. Oxid Med Cell Longev. 2019;2019:1896041. doi:10.1155/2019/1896041

22. Mokhtari V, Afsharian P, Shahhoseini M, Kalantar SM, Moini AA Review on various uses of N-acetyl cysteine. Cell J. 2017;19(1):11-17.

23. Iguchi M, Ikegami M, Kiwamoto $\mathrm{H}$, et al. Effect of verapamil on urinary calcium and oxalate excretion in renal stone formers. Hinyokika Kiyo. 1993;39(5):425-431.

24. Sarica K, Erturhan S, Altay B. Effect of verapamil on urinary stone-forming risk factors. Urol Res. 2007;35(1):23-27. doi:10.10 07/s00240-006-0075-z

25. Chen J, Cha-Molstad H, Szabo A, Shalev A. Diabetes induces and calcium channel blockers prevent cardiac expression of proapoptotic thioredoxin-interacting protein. Am J Physiol Endocrinol Metab. 2009;296(5):E1133-1139. doi:10.1152/ajpendo.90944.2008

26. Al-Gayyar MM, Abdelsaid MA, Matragoon S, Pillai BA, ElRemessy AB. Thioredoxin interacting protein is a novel mediator of retinal inflammation and neurotoxicity. Br J Pharmacol. 2011;164 (1):170-180. doi:10.1111/bph.2011.164.issue-1

\section{Publish your work in this journal}

Research and Reports in Urology is an international, peer-reviewed, open access journal publishing original research, reports, editorials, reviews and commentaries on all aspects of adult and pediatric urology in the clinic and laboratory including the following topics: Pathology, pathophysiology of urological disease; Investigation and treatment of urological disease; Pharmacology of drugs used for the treatment of urological disease. The manuscript management system is completely online and includes a very quick and fair peer-review system, which is all easy to use. Visit http://www.dovepress.com/ testimonials.php to read real quotes from published authors. 